Editorial

\title{
Bridges for a more sustainable future: joining Environmental Management for Sustainable Universities (EMSU) and the European Roundtable for Sustainable Consumption and Production (ERSCP) conferences
}

This special volume (SV) of the Journal of Cleaner Production (JCLP) evolved from the papers presented at the combined Environmental Management for Sustainable Universities (EMSU) and the European Roundtable for Sustainable Consumption and Production conference held in Istanbul, Turkey in June 2013. It was the second time the two conferences were held together and the second time that the two groups worked together to develop a single volume of the JCLP. The objective of the combined conference was to present the advances on the two broad sets of topics, 'Education for Sustainable Development' (ESD) and 'Sustainable Production and Consumption' (SPC), as well as to seek to bridge the chasm between them. The joint conferences were expertly organised by Prof. Nilgun Ciliz and her team at Bogacizi University in Istanbul.

The Joint Conference attracted over 400 participants. Contributions at the conference were organised into the following topics:

The EMSU stream:

a. Ensuring that the needs of present and future generations can be better understood and built upon, through professionals who are well versed in Sustainable Development (SD);

b. Helping make the transition to 'sustainable societal patterns', as indicated in their declarations, charters, partnerships and conferences; and

c. Empowering university leaders, students and staffs to catalyse and implement new paradigms, to help to ensure that SD is integrated systematically as the 'Golden Thread' throughout the entire university system;

\section{The ERSCP stream:}

a. Progress in sustainable production and consumption applications;

b. The evolving roles and progress in implementation of protocols, conventions and declarations locally and globally;

c. Forecasts for ecological degradation and changes in human well-being due to dramatic climate changes and how Cleaner Production of Cleaner Products can help to prevent or to slow down the advance of such changes;

d. Debates about the needed changes in policies, procedures, tools and values that are essential to move beyond the Rio +20 vacuum; e. Discussion of the types of regional sustainability indicators;

f. Debates about new decision making mechanisms for ensuring management and conservation of rural and other natural areas; and

g. Wrestling with the trade-offs between short-term costs and benefits of action vs. the longer-term costs and benefits of nonaction.

This SV of the JCLP is divided in two columns of the bridge, with a number of papers functioning as the pins and cables to connect them. The first part, that is focussed upon ESD, contains twenty-nine papers and two book reviews, covering many examples on topics such as implementation of sustainable development in higher educational institutions, stakeholder engagement and participation, campus operations, sustainability assessment and reporting, organisational change management, and curricula design, development and delivery. In spite of the examples presented in this SV stream and the major developments in the Higher Education for Sustainable Development (HESD) field there are still many challenges facing Higher Education Institutions (HEIs), and many opportunities, in theory and in practice, to further develop this discipline and to apply the lessons learnt more systematically and holistically in all curricula in all universities, worldwide.

The second part of this SV, based upon the ERSCP stream, contains thirty seven papers focussed upon SPC, covers issues such as products and processes, firms and organisations, consumers and consumption, assessment and benchmarking, innovation and design, and up-scaling, policies and institutions.

This part of the SV addresses mechanisms that facilitated successful examples of advances towards more sustainable production and consumption to be shared and scaled-up to industrial scale and in some cases to sectoral scales. The papers highlighted that action only on one side, production or consumption, produces limited results. Therefore, better understanding the inter-relationships between consumption and production are needed to help to synergistically bridge them.

Approaches, such as responsible innovation, should be at the forefront of present and future sustainability transitions, in order to help to narrow the gap between these two broad topics. Collaboration and purposeful and synergistic inter-connections 
are required to successfully implement, test, adapt and to disseminate approaches, methods and strategies in new contexts.

You as readers of this Two-Part, Special Volume are invited to build upon the wisdom contained in these articles. We look forward to your future contributions to the Journal of Cleaner Production and to future EMSU and ERSCP conferences, for example the 'Global Cleaner Production and Sustainable Consumption' conference to be held in November, 2015 Sitges, Spain in. We also wish to express a special thanks to all authors, reviewers financial contributors and others who helped to make the joint conference and this Special Volume possible.

Rodrigo Lozano* Copernicus Institute of Sustainable Development, Utrecht University, Heidelberglaan, 2, PO Box 80115, 3508TC Utrecht, The Netherlands

Nilgun Ciliz Sustainable Development and Cleaner Production Centre, Institute of Environmental Sciences, Boğaziçi University, 34342 Bebek, Istanbul, Turkey

E-mail address: cilizn@boun.edu.tr.

Tomas B. Ramos CENSE - Center for Environmental and Sustainability Research, Department of Environmental Sciences and Engineering, School of Science and Technology, Universidade Nova de Lisboa, Campus da Caparica, 2829-516 Caparica, Portugal E-mail address: tabr@fct.unl.pt.
Vincent Blok

Social Sciences Group, Wageningen University, Hollandseweg 1, 6706

KN Wageningen, The Netherlands E-mail address: vincent.blok@wur.nl.

Sandra Caeiro CENSE - Center for Environmental and Sustainability Research, School of Science and Technology, Universidade Nova de Lisboa,

Portugal

Department of Science and Technology, Universidade Aberta, Rua Escola Politecnica, $n^{\circ} 141,1269-001$ Lisbon, Portugal E-mail address: scaeiro@uab.pt.

Bernardus van Hoof School of Management, Universidad de Los Andes, Calle 21 \# 01 - 20, Bogota, Colombia E-mail address: bvan@uniandes.edu.co.

Donald Huisingh Institute for a Secure and Sustainable Environment (ISSE), University of TN, Knoxville, TN, USA E-mail address: donaldhuisingh@comcast.net.

* Corresponding author. E-mail addresses: r.lozano@uu.nl, rodlozano@orgsustainability.com (R. Lozano).

27 May 2015 Available online 5 June 2015 